\title{
Some Residually Solvable One-Relator Groups
}

\author{
KATALIN BENCSÁTH, ANDREW DOUGLAS, \\ AND DELARAM KAHROBAEI
}

\begin{abstract}
This communication records some observations made in the course of studying one-relator groups from the point of view of residual solvability. As a contribution to classification efforts we single out some relator types that render the corresponding one-relator groups residually solvable.
\end{abstract}

\section{Introduction}

It is well known that free groups are residually nilpotent and, consequently, residually solvable. The literature contains a sizable amount of information about structural, residual, virtual properties of onerelator groups. The purpose of this communication is to offer a collection of facts and examples gathered while attempting to characterize the residually solvable one-relator groups in terms (of the form) of the (single) defining relator. In what follows we prove sufficiency results for certain cases when the relator is a commutator, and then raise some questions.

The class of one-relator groups shows a varied pattern of behavior with respect to residual properties. We begin with reviewing some of the literature that motivated our interest in the topic. G. Baumslag in [3] showed that positive one-relator groups, which is to say that the relator has only positive exponents, are residually solvable. In the same paper he provided a specific example to demonstrate that not all one-relator groups are residually solvable. A free-bycyclic group is necessarily residually solvable. As well are the freeby-solvable Baumslag-Solitar groups $B_{m, n}$ (the groups with presentation $\left\langle a, b ; a^{-1} b^{m} a=b^{n}\right\rangle$ for pairs of non-zero integers $\left.m, n\right)$, by a result of Peter Kropholler [15] who showed that in these groups the second derived subgroup is free. The Baumslag-Solitar groups

Partially supported by PSC/CUNY. 
$B_{1, n}(n \neq 0$ integer $)$ are solvable but not polycyclic; and the nonHopfian (therefore not residually finite) Baumslag-Solitar groups are not residually nilpotent.

It is worth mentioning here that a large class of residually solvable one-relator groups is indicated by [8]: G. Baumslag, Fine, Miller and Troeger established that many one-relator groups, in particular cyclically pinched one-relator groups, are either free-by-cyclic or virtually free-by-cyclic. Further, a recent result of M. Sapir and I. Spakulova in [17] tells that, with probability (measured in terms of the length of the relator) tending to 1 , a one-relator group with at least 3 generators is residually finite, even virtually a residually (finite $p$ )-group, and coherent, for all sufficiently large primes $p$. In his subsequent work [16] M. Sapir also focuses on residual properties of one-relator groups with at least 3 generators. Our attention is turned mainly toward two-generator one-relator groups.

Our two main results concern the situation where $G$ is a onerelator group whose relator is a commutator. First we recall, in fair detail, some classes of one-relator groups whose behavior with regard to residual solvability has been established before. Then we show sufficiency, for residual solvability, of certain conditions imposed on the (single) defining relator of the one-relator group. Then, we provide examples illustrating the difficulty in determining residual solvability of one-relator groups with arbitrary commutator.

Clearly, attempts to find criteria for residual solvability could be facilitated by linkages to outcomes of recent and older studies on the (fully) residually freeness of one-relator groups, in particular. For example, surface groups are easily found residually solvable since they are known to be fully residually free [6]. Also, in [2] B. Baumslag shows residual freeness for one-relator groups of the type $\left\langle a_{1}, \cdots, a_{k} ; a_{1}{ }^{w_{1}} \cdots a_{k}{ }^{w_{k}}=1\right\rangle$, where $k>3$, and the $w_{i}$ 's in the ambient free group on $a_{1}, \cdots, a_{k}$ satisfy certain conditions; thus residual solvability for such one-relator groups is immediate.

\section{Preliminaries}

For convenience, we start with a list of some of the definitions, facts, and theorems we will rely on throughout.

Theorem 2.1 (Von Dyck). Suppose $G=\langle X ; R\rangle$ and $D=\langle X ; R \cup$ $S\rangle$, with presentation maps $\gamma$ and $\mu$ respectively. Then $x \mu \mapsto x \gamma$ $(x \in X)$ defines a homomorphism of $G$ onto $D$. 
Theorem 2.2 (Freiheitssatz, [14]). Let $G$ be a one-relator group, i.e., $G=\left\langle x_{1}, \cdots, x_{q} ; r=1\right\rangle$. Suppose that the relator $r$ is cyclically reduced, i.e., the first and the last letters in $r$ are not (formal) inverses of each other. If each of $x_{1}, \cdots, x_{q}$ actually appears in $r$, then any proper subset of $\left\{x_{1}, \cdots, x_{q}\right\}$ is a free basis for a free subgroup of $G$.

W. Magnus' method of structure analysis [14] for groups with a single defining relation has the following immediate consequence:

Lemma 2.3. Let $G=\langle b, x, \cdots, c ; r=1\rangle$ be a one-relator group. Suppose that $b$ occurs in $r$ with exponent sum zero and that upon reexpressing $r$ in terms of the conjugates $b^{i} x b^{-i}=x_{i}, \cdots, b^{i} c b^{-i}=c_{i}$ $(i \in \mathbb{Z})$ and renaming $r$ as $r_{0}, \mu$ and $\nu$ are respectively the minimum and maximum subscripts of $x$ occurring in $r_{0}$. If $\mu<\nu$ and if both $x_{\mu}$ and $x_{\nu}$ occur only once in $r_{0}$ then $N=g p_{G}(x, \cdots, c)$ is free. If $G$ is a two-generator group with generators $b$ and $x$, then $N$ is free of rank $\nu-\mu+1$.

Definition 2.4. A group $G$ is residually solvable if for each $w \in G$ $(w \neq 1)$, there exists a solvable group $S=S(w)$ and an epimorphism $\phi: G \longrightarrow S$ such that $w \phi \neq 1$.

Theorem 2.5 (Kahrobaei, [12], [13]). Any generalized free product of two finitely generated torsion-free nilpotent groups, amalgamating a cyclic subgroup, is an extension of a residually solvable group by a solvable group. It is therefore residually solvable.

Theorem 2.6 (Kahrobaei, [12], [13]). Any generalized free product of an arbitrary number of finitely generated nilpotent groups of bounded class, amalgamating a subgroup central in each of the factors, is an extension of a free group by a nilpotent group. It is therefore residually solvable.

Theorem 2.7 (Kahrobaei, [12], [13]). The generalized free product of a finitely generated torsion-free abelian group and a nilpotent group is (residually solvable)-by-abelian-by-(finite abelian), consequently residually solvable.

Note that the groups in all three of these theorems above satisfy the conditions of K. Gruenberg's portent observation [10] that we record here as

Lemma 2.8. Suppose $P$ is any group, $K \triangleleft P$ with $P / K$ solvable and $K$ residually solvable. Then $P$ is residually solvable. 


\section{The Single Relator is a Commutator}

We first recall a result from [4] for a particular class of non-positive one-relator groups. Let $G$ be a group that can be presented in the form,

$$
G=\left\langle t, a, \ldots, c ; u w^{-1}=1\right\rangle,
$$

where $u$ and $w$ are positive words in the given generators and each generator occurs with exponent sum zero in $u w^{-1}$. Then $G$ is residually solvable. In fact, $G$ is free-by-cyclic.

Now consider the group

$$
H=\langle t, a, \ldots, c ;[u, w]=1\rangle .
$$

If $u$ and $w$ are positive, $H$ can be recognized as one of the groups in the preceding class (1). Hence $H$ is free-by-cyclic and therefore residually solvable. However, known examples show that residual solvability for $H$ is not guaranteed once the requirement that both $u$ or $w$ be positive is relaxed:

Example 3.1. [4] If $G=\langle a, b, \ldots, c ;[u, v]=1\rangle$, where

$$
u=a, v=[a, b]\left[w, w^{b}\right], \text { and } w=[a, b]^{-1}[a, b]^{a},
$$

then $G$ is not residually solvable.

Proof. It follows from Magnus' solution of the word problem that $w \neq 1$ in $G[14]$. Since $[u, v]=1$, we find that

$$
[a, b]^{a}\left[w, w^{b}\right]^{a}=[a, b]\left[w, w^{b}\right],
$$

so that

$$
w=[a, b]^{-1}[a, b]^{a}=\left[w, w^{b}\right]\left(\left[w, w^{b}\right]^{a}\right)^{-1} .
$$

Thus $w$ lies in every term of the derived series of $G$.

In contrast, the next example is a residually solvable one-relator group.

Example 3.2. The group $G=\langle a, b ;[a,[a, b]]\rangle$ is free-by-cyclic.

Proof. We expand and re-express the relator,

$$
r=[a,[a, b]]=a^{-1}[a, b]^{-1} a[a, b]=a^{-1} b^{-1} a^{-1} b a b^{-1} a b .
$$

Observe that in

$$
r_{0}=b_{1}^{-1} b_{2} b_{1}^{-1} b_{0}
$$

$\mu=2, \nu=0, b_{0}$ and $b_{2}$ both occur only once, so we can invoke Lemma 2.3. Therefore $G$, as a cyclic extension of the free group $N=g p_{G}(b)$, is residually solvable (cf. 2.8). 


\section{Connection Between Generalized Free Products and One-Relator Groups}

Over the years since W. Magnus developed his treatment of onerelator groups the increased interest in them yielded many new results. Karrass and Solitar in 1971 showed that a subgroup of a one-relator group is either solvable or contains a free subgroup of rank two. G. Baumslag and Shalen showed that every one-relator group with at least four generators can be decomposed into a generalized free product of two groups where the amalgamated subgroup is proper in one factor and of infinite index in the other. Fine, Howie and Rosenberger [7], and Culler and Morgan [9] showed that any one-relator group with torsion and at least three generators can be decomposed, in a non-trivial way, as an amalgamated free product. These results made it seem reasonable to expect that a closer look at the residual solvability of generalized free products of two groups could provide further tools for detection of residual solvability of one-relator groups. The following result confirms that assumption.

Theorem 4.1. The group $G=\langle a, b ;[a, w]\rangle$, where $w=[a, b]^{n}(n \in$ $\mathbb{N})$, is residually solvable.

Proof. Put $N=g p_{G}(b)$, the normal closure of $b$ in $G$. Using the Magnus break-down, we consider:

$$
N_{0}=\left\langle b_{0}, b_{1}, b_{2} ;\left(b_{1} b_{0}\right)^{n}=\left(b_{2}^{-1} b_{1}\right)^{n}\right\rangle .
$$

Now let

$$
x_{0}=b_{1}^{-1} b_{0}, x_{1}=b_{2}^{-1} b_{1}, y=b_{1} .
$$

Tietze transformations confirm that

$$
N_{0}=\left\langle x_{0}, x_{1}, y ;\left(x_{0}\right)^{n}=\left(x_{1}\right)^{n}\right\rangle=\left\langle x_{0}, x_{1} ;\left(x_{0}\right)^{n}=\left(x_{1}\right)^{n}\right\rangle *\langle y\rangle .
$$

Next let $K=\left\langle x_{0}, x_{1} ;\left(x_{0}\right)^{n}=\left(x_{1}\right)^{n}\right\rangle$. Clearly

$$
K=\left\{\left\langle x_{0}\right\rangle *\left\langle x_{1}\right\rangle ;\left\langle x_{0}^{n}\right\rangle=\left\langle x_{1}^{n}\right\rangle\right\}
$$

Since each factor of $K$ is abelian, by Theorem $2.5 K$ is residually solvable. The free factor of $N_{0},\langle y\rangle$ is also residually solvable. Therefore $N_{0}$ is residually solvable, and it follows for every $i \in \mathbb{N}$ that $N_{i}$ is residually solvable. If we put $N_{i, j}=g p\left(N_{i}, N_{i+1}, \ldots, N_{j}\right)$, the preceding approach gives

$N_{i, j}=\left\langle x_{i}\right\rangle *\left\langle x_{i}^{n}\right\rangle=\left\langle x_{i+1}^{n}\right\rangle *\left\langle x_{i+1}\right\rangle * \cdots *\left\langle x_{j}\right\rangle *\left\langle x_{j}^{n}\right\rangle=\left\langle x_{j+1}^{n}\right\rangle *\left\langle x_{j+1}\right\rangle *\langle y\rangle$. 
Therefore, by Theorem 2.6, $N_{i, j}$ is residually solvable. A task that remains for completing the proof is to show that the ascending union $N=\cup_{r<0 ; s>0} N_{r, s}$ is residually solvable, which will be taken care of by the following Proposition 4.2. Granted that, the residual solvability of $G$ follows with the use of Corollary 2.8.

Proposition 4.2. $N=\cup_{r<0 ; s>0} N_{r, s}$ is residually solvable.

Proof. We will retain notation from the proof of Theorem 4.1 and start with the assumption that $N_{i, j}$ is residually solvable for all $i, j \in$ $\mathbb{N}(i \leq j)$. For the derived series of $N=\cup_{r<0 ; s>0} N_{r, s}$, we have

$$
\delta_{i} N=\delta_{i}\left(\cup_{r<0 ; s>0} N_{r, s}\right) .
$$

Every element $g \in \delta_{i} N$ is a finite product of commutators of elements from a (finite) subset of the $N_{r, s}$ groups. So $g \in \delta_{i} N_{r, s}$ for suitably small value of $r<0$ and suitably large value of $s>0$. Thus $\delta_{i} N=$ $\cup_{r<0 ; s>0} N_{r, s}$. Now, if $j, k$ are a pair of fixed integers the infinite union above can be rewritten as

$$
\delta_{i} N=\cup_{r<0 ; s>0} \delta_{i} N_{j+r, k+s},
$$

so that,

$$
\delta_{i} N \cap N_{j, k}=\left(\cup_{r<0 ; s>0} \delta_{i} N_{j+r, k+s}\right) \cap N_{j, k} .
$$

Equivalently,

$$
\delta_{i} N \cap N_{j, k}=\cup_{r<0 ; s>0}\left(\delta_{i} N_{j+r, k+s} \cap N_{j, k}\right) .
$$

Further, each term in the union can be written as

$$
\delta_{i} N_{j+r, k+s} \cap N_{j, k}=\left(\delta_{i} N_{j, k+s} \cap N_{j, k}\right) \cap\left(\delta_{i} N_{j+r, k} \cap N_{j, k}\right) .
$$

And because $s<0$ and $r>0$, an argument fashioned after that in [3, p. 175, Lemma 4.3] yields after suitable conjugations that

$$
\begin{gathered}
\delta_{i} N_{j, k+s} \cap N_{j, k}=\delta_{i} N_{j, k} \text { and } \\
\delta_{i} N_{j+r, k} \cap N_{j, k}=\delta_{i} N_{j, k} .
\end{gathered}
$$

So each term in the union can be re-expressed as

$$
\delta_{i} N_{j+r, k+s} \cap N_{j, k}=\delta_{i} N_{j, k} .
$$

Notice that this expression is independent of $r$ and $s$. Thus, we get

$$
\delta_{i} N \cap N_{j, k}=\delta_{i} N_{j, k}([3, \text { pg. 175, line 16]). }
$$

We claim that

$$
\delta_{i} N \cap N_{j, k}=\delta_{i} N_{j, k}
$$


implies that $N$ is residually solvable. To see this, let $g$ be a nontrivial element of

$$
N=\cup_{r<0 ; s>0} N_{r, s} .
$$

Then there is an integer $j=j(g) \in \mathbb{N}$ such that $g \in N_{-j, j}$. By our (inductive) hypothesis at the outset $N_{-j, j}$ is residually solvable. Consequently, there exists an integer $i \in \mathbb{N}$ such that $g \notin \delta_{i} N_{-j, j}$. Then, since $\delta_{i} N \cup N_{-j, j}=\delta_{i} N_{-j, j}$, we see that $g \notin \delta_{i} N \cap N_{-j, j}$. But $g \in N_{-j, j}$. So it must be the case that $g \notin \delta_{i} N$. Thus we have found a normal subgroup $\delta_{i} N \triangleleft N$ with the property that $g \notin \delta_{i} N$ and $N / \delta_{i} N$ is solvable. Hence $N$ is residually solvable.

\section{The Relator is a Basic Commutator}

The tools of the Magnus theory were of good use for proving residual solvability through gaining information about the structure of the two-generator one-relator groups where the relator is a particular type of basic commutator.

We begin with recalling P. Hall's [11] definition of the basic commutators (in terms of the free group $F$ on $\left\{x_{1}, \ldots, x_{q}\right\}$ ) and their linear ordering (in terms of their weights).

Definition 5.1. Basic Commutators.

(1) The basic commutators of weight one with their linear order are $x_{1}<x_{2}<\cdots<x_{q}$; for their weights we write $w t\left(x_{i}\right)=1$.

(2) Having defined the basic commutators of weight less than $n$, the basic commutators with weight $n$ are of the form $c_{n}=\left[c_{i}, c_{j}\right]$ where $c_{i}$ and $c_{j}$ are all the basic commutators satisfying $w t\left(c_{i}\right)+w t\left(c_{j}\right)=n, c_{i}>c_{j}$, and such that if $c_{i}=\left[c_{s}, c_{t}\right]$, then $c_{j} \geq c_{t}$.

In the following we will use the notation $s_{1}=x$ and $s_{k+1}=\left[s_{k}, y\right]$ for positive integers $k$.

Theorem 5.2. The group $G=\left\langle x, y ; r=\left[s_{k}, y\right]\right\rangle$ is free-by-cyclic, therefore residually solvable.

Proof. Following the Magnus theory we put $x_{i}=y^{-i} x y^{i}$ for this two generator case. Using induction on the weight of the commutator and the relationship

$$
\left[s_{k}, y\right]=s_{k}^{-1}\left(s_{k}\right)^{y}(k>0),
$$


we see that the minimum index and maximum index in $r_{0}$ are 0 and $k$, respectively, and both of $x_{0}$ and $x_{k}$ occur only once in $r_{0}$. By Lemma 2.3 it follows, similarly to previous cases, that $G$ is free-bycyclic.

\section{Open Problems}

(1) Is it algorithmically decidable whether a one-relator group is residually solvable?

(2) Are one-relator groups generically residually solvable? In other word, are they in most cases residually solvable? I. Kapovich conjectured [5] that many one-relator groups are finitely generated freeby-cyclic.

(3) Do there exist residually finite one-relator groups that are not residually solvable? (This is recasting a question in [1] in this context.)

(4) As defining relators, certain basic commutators were shown in this paper to render the respective one-relator groups residually solvable. Would all basic commutators have that property? If not, can the techniques used here be extended to one-relator groups with further types of basic commutator for their defining relator?

(5) Find further examples of non-positive one relator groups that fail to be residually solvable.

(6) Find examples of residually solvable one-relator groups that are not free-by-cyclic.

\section{REFERENCES}

[1] G. Arzhantseva, P. de la Harpe, D. Kahrobaei, Z. Sunik, The true prosoluble completion of a group: Examples and open problems, Journal of Geometriae Dedicata, Springer Netherlands 124 (2007) No.1, 5-26.

[2] B. Baumslag, Residually free groups, Proc. London Math. Soc. (3) 17 (1967) $402-18$.

[3] G. Baumslag, Positive one-relator groups, Trans. Amer. Math. Soc. 156 (1971), 165-183.

[4] G. Baumslag, Free subgroups of certain one-relator groups defined by positive words, Math. Proc. Cambridge Phil. Soc. 93 (1983), 247-251.

[5] O. Bogopolski, A. Martino, O. Maslakova, and E. Ventura, The conjugacy problem is solvable in free-by-cyclic groups, Bull. London Math. Soc. 38 (2006), 787-794.

[6] C. Champetier, V. Guirardel, Limit groups as limits of free groups, Israel J. Math. 146 (2005), 1-75.

[7] B. Fine, J. Howie, G. Rosenberger, One-relator quotients and free products of cyclics, Proc. Amer. Math. Soc. 102 (1988), 249-254. 
[8] G. Baumslag, B. Fine, C.F. Miller, and D. Troeger, Virtual properties of cyclically pinched one-relator groups, Int. J. Algebra and Computation 19 (2009), 213-227.

[9] M. Culler, J. Morgan, Group actions on R-trees, Proc. London Math. Soc. (3) 55 (1987), 571-604.

[10] K.W. Gruenberg, Residual Properties of Infinite Soluble Groups, Proc. London Math. Soc. (3) 7 (1957), 29-62.

[11] M. Hall, The theory of groups. Chelsea Publishing Company, 1976.

[12] D. Kahrobaei, Residual solvability of generalized free products, The City University of New York (GC) PhD Thesis, October 2004.

[13] D. Kahrobaei, On residual solvability of generalized free products of finitely generated nilpotent groups, Comm. in Algebra, to appear.

[14] W. Magnus, A. Karrass, and D. Solitar, Combinatorial group theory, Dover, 1975.

[15] P. H. Kropholler, Baumslag-Solitar groups and some other groups of cohomological dimension two, Comment. Math. 65 (1990), 547-558.

[16] M. Sapir, Residual properties of 1-relator groups arXiv:1001.2829, (2010).

[17] M. Sapir, I. Spakulova, Almost all one-relator groups with at least three generators are residually finite. arXiv:0809.4693 (2009).

Katalin Bencsáth,

Department of Mathematics and Computer Science,

Manhattan College,

Riverdale, New York 10471, USA

katalin.bencsath@manhattan.edu

Andrew Douglas,

Department of Mathematics,

New York City College of Technology, CUNY,

300 Jay Street, Brooklyn,

New York 11201, USA

adouglas@citytech.cuny.edu

Delaram Kahrobaei,

Doctoral Program in Computer Science Department,

CUNY Graduate Center, City University of New York,

365 Fifth Ave.,

New York 10016, USA

dkahrobaei@gc.cuny.edu

Received on 30 April 2010. 